\title{
Das Pferd im Verbrauchsgüterkaufrecht
}

\author{
Kai Bemmann
}

Rechtsanwaltskanzlei, Verden/Aller

Der deutsche Gesetzgeber hat mit Wirkung zum 01.01.2002 das Verbrauchsgüterkaufrecht im BGB installiert und dabei für die Verkäufe von einem Unternehmer an einen Verbraucher ohne Rücksicht auf den Kaufgegenstand einen besonderen Verbraucherschutz geschaffen. Gleichzeitig hat er jegliche viehkaufrechtlichen Sonderregelungen aufgehoben. Dies war nicht nur die grundlegendste Änderung des BGB seit seinem Inkrafttreten, sondern auch das Ergebnis eines überhasteten Gesetzgebungsverfahrens (Schermeier und Saenger 2003), nachdem unter Zeitdruck u. a. die Verbrauchsgüterkaufrichtlinie der EU (RL 1999/44/EG) in das deutsche Recht umgesetzt werden musste und zugleich mit ehrgeizigen politischen Zielen die Schuldrechtsreform zur Modernisierung des Veriährungs-, Leistungsstörungs- und Kaufrechts einhergehend mit der Integration der Verbraucherschutzgesetze in das BGB vollzogen werden sollte (Däubler-Gmelin 2001).

\section{Gesetzessituation in Deutschland und Europa}

Das Fehlen jeglicher viehkaufrechtlicher Sonderregelungen war im Wesentlichen von zwei Motiven getragen. Einerseits wurde aufgrund des Wortlauts der Verbrauchsgüterkaufrichtlinie kein Raum für viehkaufrechtliche Sonderregelungen gesehen. Die Richtlinie sieht keine Ausnahme für das Vieh als Kaufsache vor, so dass der deutsche Gesetzgeber sich in der Gefahr einer nicht vollständigen Umsetzung der Verbrauchsgüterkaufrichtlinie wähnte, falls er Sonderregelungen in den verbrauchsgüterkaufrechtlichen Bestimmungen des BGB vorgesehen hätte. Das wäre mit dem vermeintlichen Risiko verbunden gewesen, vor dem EuGH gegenüber einem betroffenen Verbraucher in einem Verfahren wegen nicht richtlinienkonformer Gesetzgebung zu unterliegen. Es ist allerdings fraglich, ob dem europäischen Gesetzgeber bei Schaffung seines Rechtaktes überhaupt bewusst war, dass er elementar in die wirtschaftlichen Belange der Tierzucht eingreift.

Andererseits glaubte der deutsche Gesetzgeber in Ansehung des Fortschritts der tiermedizinischen Wissenschaft, ohnehin keine Sonderregelungen für Vieh mehr zu benötigen. Er war sich somit ansatzweise bewusst, dass auch der Viehhandel der Verbrauchsgüterkaufrichtlinie unterfallen könnte und dies ein Anlass zur kompletten Aufhebung des Viehkaufrechts sein sollte (Schmidt-Räntsch 1998). Der Gesetzgeber glaubte, dass eine solche Regelung problemlos sei. Denn die Tiermedizin sei in der Lage, alle körperlichen Mängel von Tieren hinsichtlich ihres Entstehungszeitraumes retrospektiv zu bewerten (BT-Drs. 14/6040). Letzteres ist mit Sicherheit falsch. Der Gesetzgeber hat erstmals in einem Gesetzgebungsverfahren ohne tiermedizinische und/oder landwirtschaftliche Beratung
Hand an Viehkaufgesetze gelegt und sich auf bloße MutmaBungen weniger Referenten verlassen (Bemmann 2003), weil schon aus zeitlichen Gründen mit Vertretern aus Wissenschaft und Praxis nur unzureichend diskutiert werden konnte (Schulze und Schulte-Nölke 2001). Dadurch hat der Gesetzgeber die Rechtsprechung und die Tiermedizin vor nicht lösbare Aufgaben gestellt. Denn die Tiermedizin kann den Anforderungen des Gesetzgebers nicht gerecht werden. Sie hat ernorme Fortschritte hinsichtlich der Diagnostik und Behandlung sowie in der Technik genommen. Sie ist jedoch regelmäßig nicht in der Lage, Erkrankungen hinsichtlich ihres Entstehungszeitpunktes überhaupt und dann auch noch über mehrmonatige Zeiträume zu bewerten (Eversfield 2004; Bemmann 2003), zumal dies auch nicht ihr erstrangiges Ziel ist. Sie hat sich vielmehr primär der Heilbehandlung und nicht dem Dienst des Gesetzgebers verschrieben, der es erstmals nicht für notwendig erachtet hat, die namhaften Vertreter der tiermedizinischen Wissenschaft anzuhören. Infolgedessen bleibt es der Rechtsprechung überlassen, nach Beweislastregeln zu entscheiden.

Aber auch die Missachtung viehkaufrechtlicher Belange bei Schaffung des europäischen Rechtsaktes stößt auf Bedenken, die im Ergebnis Zweifel an der Rechtswirksamkeit der Verbrauchsgüterkaufrichtlinie und den auf ihr beruhenden Kaufrechtsregelungen der Mitgliedsstaaten begründen. Zunächs† einmal ist auffällig, dass zumindest die Mitgliedsstaaten Österreich, Spanien und Portugal die Verbrauchsgüterkaufrichtlinie anscheinend als ein Konzept verstanden haben, das allein dem Schutz des nicht mit technischen Kenntnissen ausgestatteten Verbrauchers vor fehlerhafter Fabrikationsware dienen soll. Sie haben jedenfalls für die Kaufsache "Vieh" Sonderregelungen geschaffen. Die Österreicher räumen lediglich hinsichtlich der Fristen dem Verbraucher eine Besserstellung gegenüber dem Unternehmer ein und haben im Übrigen ihre viehkaufrechtlichen Sonderregelungen beibehalten (Reischaver 2002).

Noch weitgehender erscheinen die spanischen und portugiesischen Sonderregelungen, die weiterhin auf Verordnungen verweisen und im Übrigen Ausnahmen für Viehmärkte sowie kurze Fristen und Verweise auf Verkehrssitten enthalten (Schermaier und Saenger 2003).

Es sind aber nicht vornehmlich die bürgerlich rechtlichen Regelungen einzelner Mitgliedsstaaten, die Bedenken gegen die Art der Umsetzung der Verbrauchsgüterkaufrichtlinie in deutsches Recht verursachen. Vielmehr hat die EU im eigenen Gesetzgebungsverfahren wahrscheinlich gar nicht erkannt, dass sie mit ihren Verbraucherschutzregeln auch die Tierzucht erfasst und dabei in deren wirtschaftliche Belange mit existen- 
ziellen Folgen eingreift; oder der Rechtsakt sollte entsprechend seinem konzeptionellen Zuschnitt nur dem Handel mit industriell gefertigten Waren gelten und es ist in Anbetracht des für jedermann deutlich erkennbaren Gesetzeszwecks die ausdrückliche Klarstellung versäumt worden, dass der Handel mit Tieren und Naturprodukten der RL nicht unterliegt. Denn es ist nicht vorstellbar, dass die EU wissentlich verfahrensfehlerhaft vorgegangen sein könnte. Der europäische Gesetzgeber hatte nämlich eigentlich schon vor Jahrzehnten erkannt, dass die Tierproduktion rechtlich anders beurteilt werden muss, als die Herstellung lebloser Waren. Deshalb hatte er bereits 1977 mit der Einsetzung des ständigen Tierzuchtausschusses beschlossen, dass ein mit sachverständigen Vertretern der Mitgliedsstaaten besetzter Ausschuss an den Gesetzgebungsverfahren immer dann zu beteiligen ist, wenn die Belange der Tierzucht von den Gemeinschaftsregelungen tangiert werden sollen (77/505/EWG). Diese Beteiligungsnotwendigkeit ist übersehen worden, so dass die Verbrauchsgüterkaufrichtlinie in Kraft trat, ohne dass ihre Auswirkungen auf den Verkauf von Tieren im Gesetzgebungsverfahren beraten werden konnte. Als der deutsche Gesetzgeber spätestens im Anhörungsverfahren aufgrund der Stellungnahmen der Tierzuchtverbände (Pelhak 2001) und kundiger Juristen (Adolphsen 2001) erkannt hatte, dass das geplante Verbrauchsgüterkaufrecht für den Viehhandel ungeeignet sein könnte, kam ein Umdenken unabhängig von der dazu fehlenden Bereitschaft schon deshalb nicht mehr in Betracht, weil die Frist zur Anfechtung der Verbrauchsgüterkaufrichtlinie gem. Art. 230 EGV bereits lange verstrichen war. Also musste der deutsche Gesetzgeber die Richtlinie umsetzen und litt dabei unter dem bereits geschilderten Zeitdruck und seinem politischen Ehrgeiz.

Das Versäumnis des deutschen Gesetzgebers, die Verbrauchsgüterkaufrichtlinie gem. Art. 230 EGV anzufechten, darf sich allerdings nicht für den betroffenen Unternehmer in dessen individuellem Rechtsstreit auswirken. Denn dieser war am europäischen Gesetzgebungsverfahren nicht beteiligt und konnte kein Anfechtungsrecht ausüben. Sonst könnte sich ein unwirksamer europäischer Rechtsakt nun infolge seiner unkritischen Umsetzung in innerstaatliches Recht gegenüber dem Unternehmer in einem Zivilrechtsstreit nachteilig auswirken. Jeder betroffene Unternehmer hat deshalb in seinem individuellen Rechtsstreit trotz der für den deutschen Staat abgelaufenen Anfechtungsfrist die Möglichkeit, die Nichtigkeitseinrede gegenüber der Verbrauchsgüterkaufrichtlinie und den daraus umgesetzten verbrauchsgüterkaufrechtlichen Vorschriften des BGB zu rügen, sobald er feststellt, dass ein deutsches Gericht diese Vorschriften zu seinem Nachteil in einem Viehkaufrechtsstreit anwenden wird. Diese Rüge muss zur Folge haben, dass das deutsche Gericht den Rechtsstreit zur Überprüfung einer Nichtigkeit der Verbrauchsgüterkaufrichtlinie gem. Art. 234 i. V. m. Art. 241 EGV dem Europäischen Gerichtshof zur Entscheidung vorlegen muss. Eine solche Vorlegung kommt in erster Linie in pferdekaufrechtlichen Streitigkeiten in Betracht. Denn der Pferdehandel ist dadurch gekennzeichnet, dass der Verkauf vom Unternehmer an den Verbraucher von großer volkswirtschaftlicher Bedeutung ist, während der übrige Viehhandel dadurch bestimmt wird, dass der Landwirt, Viehhändler oder die Lebensmittelindustrie Handel untereinander betreiben. Dies führt dazu, dass jeweils Unternehmer Verträge miteinander schließen, die dann nicht den verbrauchsgüterkaufrechtlichen Beschränkungen unter- liegen. Außerdem ist die viehkaufrechtliche Bedeutung der Pferde allein schon deshalb größer, weil diese Tiere aufgrund ihrer Lebenserwartung die Chance haben, die übliche Prozessdaver zu überleben.

\section{Wirtschaftliche Bedeutung des Pferdehandels}

Nach den Berichten seriöser deutscher Presseorgane hat der Pferdehandel eine erhebliche volkswirtschaftliche Bedeutung. Die Jahresumsätze im Pferdehandel sollen danach in Deutschland mindestens 1 Milliarde Euro (Stampf und Stuppe 2002), nach der Ipsos-Studie sogar 5 Milliarden Euro betragen (Kreling 2004). Außerdem beschäftigt der deutsche Pferdehandel 300.000 Menschen, nämlich ebenso viele wie der Volkswagenkonzern (Fuchs 2004). Eine ähnliche Situation besteht im nahen EU-Ausland. So bietet der Pferdehandel in den Niederlanden 12000 Menschen Arbeit und trägt zu einem Jahresumsatz von 1,3 Milliarden Euro bei. Die Bedeutung eines solchen Umsatzes wird erst dann richtig deutlich, wenn man sich vergegenwärtigt, dass das Blumenland Holland im Blumenhandel "nur" 500 Millionen Euro Jahresumsatz schreibt (Kreling 2004). In Schweden übersteigt die Wirtschaftskraft der Pferdeindustrie sogar die weltweiten Umsätze des bedeutendsten schwedischen Wirtschaftsunternehmens IKEA um ein vielfaches (Kamph 2004).

Die Bedeutung für die Volkswirtschaft und den Arbeitsmarkt waren dem Gesetzgeber mit Sicherheit nicht bewusst, als er die viehkaufrechtlichen Sonderregelungen aufhob, denn niemand will ihm unterstellen, dass er in der heutigen Wirtschaftslage durch Gesetze bewusst bedeutende Zweige der Volkswirtschaft schwächen will. Er hat schlichtweg nicht erkannt, dass sich Vieh als Objekt des Verbraucherschutzes schon aus tatsächlichen Gründen nicht eignet, weil der Züchter von Vieh im Gegensatz zum Produzenten der Fabrikationsware es nicht beeinflussen kann, welche Entwicklung das Tier im Laufe seines Lebens nimmt und welchen Umwelteinflüssen es ausgesetzł wird. Insbesondere nimmt der Züchter keinen Einfluss auf die Beschaffenheit des Viehs. Vielmehr konstruiert die Natur nach ihren "Gesetzen" und es liegt kein von finanzkräftigen Wirtschaftsunternehmen vorgegebener Konstruktionsplan vor. Deshalb ist ein verschärfter Schutz des Käufers einhergehend mit einer Benachteiligung des Viehhandels von vornherein schon aus tatsächlichen Gründen nicht zu rechtfertigen. Unabhängig davon ist starke Kritik an der derzeitigen Gesetzgebung im Allgemeinen (Redeker 2004) und an dem gesamten Schuldrechtsmodernisierungsgesetz (Dauner-Lieb 2004) im Besonderen allgegenwärtig. Fehlerbehaftete, realitätsfremde Gesetze stellen die Rechtsprechung immer wieder vor die schwierige Aufgabe, diese Gesetze durch juristische Kunstgriffe so zu korrigieren, dass sie der Lebensrealität gerecht werden. Es soll deshalb untersucht werden, ob und in welcher Weise die Rechtsprechung einen Ausweg aus der unpassenden Gesetzessituation finden kann, die seit der Anwendung des Verbrauchsgüterkaufrechts auf den Viehhandel besteht. Derzeit zeigen sich schon Probleme im Umgang mit dem Unternehmertatbestand und in der Abgrenzung einer negativen Beschaffenheitsvereinbarung vom Haftungsausschluss. Diese betreffen allerdings das Verbrauchsgüterkaufrecht im Allgemeinen. In viehkaufrechtlichen Streitigkeiten zeigen sich insbesondere drei typische verbrauchsgüterkaufrechtliche Problemkreise: 
Wann liegt der Verkauf eines gebrauchten Tieres im Wege öffentlicher Versteigerung vor, so dass die verbrauchsgüterkaufrechtlichen Regelungen generell nicht anzuwenden sind (§ 474 Abs. 1 Satz 2 BGB)?

Ist die verbrauchsgüterkaufrechtliche Beweislastumkehrregelung mit dem Vieh als Art der Kaufsache unvereinbar (§ 476 $\mathrm{BGB})$ ?

Ist die verbrauchsgüterkaufrechtliche Beweislastumkehrregelung mit Tiermängeln unvereinbar (§ 476 BGB)?

Die Rechtsprechung hat sich mit diesen Problemkreisen bereits befasst.

\section{Verkauf eines gebrauchten Pferdes in öffentlicher Ver- steigerung}

Die Tatbestandsmerkmale des "Gebrauchtseins" und der "Öffentlichen Versteigerung" unterliegen Definitionsproblemen.

\section{Öffentliche Versteigerung}

Der Gesetzgeber hat die öffentliche Versteigerung gebrauchter Sachen den Haftungsverschärfungen des Verbrauchsgüterkaufrechts entzogen, solange der Verbraucher freien Zugang hat. Die Definition der öffentlichen Versteigerung i. S. d. Verbrauchsgüterkaufrechts bereitet offensichtlich Schwierigkeiten. Der Gesetzgeber hatte eine solche Ausnahme zunächst gar nicht vorgesehen. Schließlich ist sie dann durch den Ausschuss eingefügt worden, weil sie bei der Versteigerung von Fundsachen nützlich sein könnte (BT-Drs. 14/7052).

Das Tatbestandsmerkmal der öffentlichen Versteigerung war im BGB bereits vielfach und auch schon in den viehkaufrechtlichen Sonderregelungen enthalten. So sah nämlich die Verfahrensvorschrift für das einstweilige Verfügungsverfahren gem. $\S 489$ BGB a. F. eine Verwertung des Prozesstieres im Wege öffentlicher Versteigerung vor, sobald das Tier nicht mehr zu Beweiszwecken benötigt wurde. Die bisher im BGB geregelten öffentlichen Versteigerungen waren stets amtliche Versteigerungen im Sinne von $\S 383$ Abs. 3 BGB, die durch den zuständigen Gerichtsvollzieher oder einen anderen befugten Beamten, z. B. einen Notar oder aber durch einen "öffentlich angestellten Versteigerer" zu erfolgen haben. Letzterer kann entgegen dem Wortlaut des Gesetzes auch ein freiberuflich tätiger Auktionator sein, sofern er die Versteigerererlaubnis gem. $\S$ 34 b Abs. 5 GewO besitzt, nämlich öffentlich bestellt und vereidigt ist (Dennhardt 2003; Wenzel 2004; Heinrichs 2005). Somit kann ein gebrauchtes Pferd durch einen öffentlich bestellten und vereidigten Auktionator unter Befreiung von den Beschränkungen des Verbrauchsgüterkaufrechts veräußert werden. Dies hat der Gesetzgeber auf eine bereits vor Inkrafttreten des SMG gehaltene offizielle Anfrage der Deutschen Reiterlichen Vereinigung ausdrükklich bestätigt (Bemmann 2003). Folglich ist der in den Motiven des Gesetzgebers vorgenommene Bezug auf die Versteigerung von Fundsachen nicht als Beschränkung, sondern beispielhaft zu verstehen.
Der Gesetzgeber hat allerdings die Formulierung des $\S 474$ Abs. 1 S. 2 BGB in Umsetzung von Art. 1 Abs. 3 der VerbrGKRL geschaffen. Deshalb wird nicht ausschließlich auf eine öffentliche Versteigerung, sondern auf eine solche, an der der Verbraucher persönlich teilnehmen kann, verwiesen. Somit ist die Frage berechtigt, ob es sich hierbei um ein neues Tatbestandsmerkmal handelt, welches gar nicht im Lichte von $\S 383$ Abs. 3 BGB, sondern anhand der Vorgaben der VerbrGKRL zu prüfen ist. Diese stellt maßgeblich auf die Möglichkeit der persönlichen Teilnahme des Verbrauchers ab. Danach kommt es also auf die freie Zugangsmöglichkeit an. Daran anknüpfend wird daher die Auffassung vertreten, dass die öffentliche Versteigerung im Sinne von $\S 474$ Abs. 1 S. 2 $B G B$ gar nicht zwingend eine amtliche Versteigerung im Sinne von $\S 383$ Abs. 3 BGB sei, die zumindest durch einen öffentlich bestellten und vereidigten Auktionator vorzunehmen ist. Vielmehr sei es eine besondere Vertriebsmethode mit der Folge, dass alle Auktionen mit freier Zugangsmöglichkeit für einen Sachmangelhaftungsausschluss privilegiert sind (Vieweg und Vollkommer 2003; Wertenbruch 2004). Diese Auffassung wird allerdings bisher weder nennenswert von der gängigen Kommentarliteratur (Faust 2003; Lorenz 2004; Heinrichs 2005) noch der Rechtsprechung (LG Münster 2003; LG Oldenburg 2004; a. A. LG München I 2004) geteilt. Denn der deutsche Gesetzgeber hat, wenn auch unbewusst, durch die gesetzliche Definition des Tatbestandsmerkmals der öffentlichen Versteigerung in § 383 Abs. 3 BGB die Ausnahmen von den verbrauchsgüterkaufrechtlichen Schutzvorschriften strenger formuliert, als dies die VerbrGKRL zugelassen hätte. Diese kennt keine Legaldefinition der öffentlichen Versteigerung, so dass sie im Wege der wörtlichen Auslegung des Richtlinientextes alle Versteigerungen privilegieren wollte, die der Verbraucherschaft öffentlich zugänglich sind. Die Übererfüllung des in der RL vorgegebenen Verbraucherschutzes beeinträchtigt die Wirksamkeit der innerstaatlichen Gesetzgebung nicht, weil die RL den Mindestmaßstab und nicht die Höchstgrenze festlegt. Also gilt $\S$ 383 Abs. 3 BGB für die Versteigerung im Sinne von $\S 474$ Abs. 1 S. 2 BGB und die Auktion ist mindestens von einem öffentlich bestellten und vereidigten Versteigerer durchzuführen, um eine "öffentliche Versteigerung" zu sein, die dann dem Verbraucher zusätzlich noch die freie Zugangsmöglichkeit gewährleisten muss.

\section{Gebrauchtes Pferd}

Die VerbrGKRL enthält keine Ausnahme für Tiere, so dass auch das Verbrauchsgüterkaufrecht des BGB trotz des Verfassungsranges des Tierschutzes in Art. 20 a GG keine Sonderregelungen für Tiere enthält. Vielmehr sind diese gem. $\S 90$ a BGB den Sachen ausdrücklich gleichgesetz (Adolphsen 2001). Deshalb wundert es auch nicht, dass der Gesetzgeber ohne Bedenken schon in den Motiven ausdrücklich eine Unterscheidung zwischen "neven" und "gebrauchten" Tieren forderte (BT-Drs. 14/6040). Eine Legaldefinition für das Gebrauchtsein hat der Gesetzgeber unterlassen, weil er glaubte, dass die Rechtsprechung bereits die Kriterien einer "neu hergestellten" Sache herausgearbeitet habe. Daran anknüpfend sollten wohl die Kriterien für das Gebrauchtsein ermittelt werden. Diese Aufgabe übertrug der Gesetzgeber ausdrücklich der Rechtsprechung. 
Dabei hat der Gesetzgeber übersehen, dass er durch eine solche Maßnahme eine lange Phase der Rechtsunsicherheit geschaffen hat, die bis zum Vorliegen höchstrichterlicher und einheitlicher Rechtsprechung andavert. Denn das Tatbestandsmerkmal des "Gebrauchtseins" gab es im BGB nicht. Die spärlich veröffentlichte Rechtsprechung zum "neu hergestellten" Tier befasste sich mit der Inhaltskontrolle von allgemeinen Geschäftsbedingungen bzw. Formularverträgen im Licht von $\S 11$ Ziff. 10 AGBG bzw. jetzt $\S 309$ Ziff. 8 b BGB, wonach in Vertragsbestimmungen über die Lieferung "neu hergestellter" Tiere zahlreiche Haftungsbeschränkungen unwirksam sind. Dabei hatte die Rechtsprechung nie zu prüfen, wann das "Neuhergestelltsein" endet und das "Gebrauchtsein" beginnt. Vielmehr beschäftigte sich die Rechtsprechung nur damit, ob noch ein "Neuhergestelltsein" vorliegt oder eine analoge Anwendung auf bestimmte Kaufsituationen von Tieren angemessen erscheint (BGH 1986; LG Aschaffenburg 1990). Diese in den Motiven des Gesetzgebers ausdrücklich erwähnte Rechtsprechung hat klargestellt, dass es sich um Einzelfallentscheidungen handelt. Sie hat bewusst offen gelassen, ob eine ähnliche Behandlung anderer Tiere, insbesondere von Pferden und weiteren Nutztieren, geboten sei (BGH 1986). Der Gesetzgeber unterlag also falschen Vorstellungen, als er davon ausging, dass die vorhandene Rechtsprechung Kriterien für den Beginn des "Gebrauchtseins" eines Tieres liefern könnte.

Indessen liefert die Rechtsprechung zum Handels-, Steuer- und Investitionszulagenrecht solche Kriterien. Denn dort spielen das Herstellungsende und der Beginn des Gebrauchtseins eine entscheidungserhebliche Rolle, weil mit dem Herstellungsende die Abschreibungsmöglichkeit für Wirtschaftsgüter des beweglichen Anlagevermögens beginnt. Dabei kommt die Rechtsprechung zu unterschiedlichen Zeitpunkten des Herstellungsendes. Beim männlichen Zuchttier endet die Herstellung mit Beginn der Zuchteinsatzmöglichkeit, während es beim weiblichen Zuchttier die Vollendung der ersten Geburt ist (BFH 1989; BFH 1997; OVG Schleswig 2004). Beim Reitpferd endet die Herstellungsphase mit dem Beginn des Zureitens und beim Turnierpferd durch den ersten Turnierstart (BFH 1981). Das Handels-, Stever- und Investitionszulagenrecht beschäftigt sich allerdings ausschließlich unter Kostengesichtspunkten mit dem Herstellungsende und dem Beginn des Gebrauchs. Im Sachmangelhaftungsrecht soll aber wegen der mit dem Gebrauch einhergehenden, erhöhten Beschädigungsrisiken zwischen "neu" und "gebraucht" unterschieden werden. Deshalb ist die Übertragung der Kriterien zum Herstellungsende unter Kostengesichtspunkten auf das Sachmangelhaftungsrecht denkbar ungeeignet. Dies gilt umso mehr, als unter Kostengesichtspunkten mehrere Herstellungsendzeitpunkte denkbar sind. So könnte z. B. eine Stute aufgrund einer Mehrfachnutzung als Sportpferd und Zuchtstute in einem Lebewesen unterschiedliche Herstellungsendzeitpunkte vereinen (Grimm 2004).

Dem Gesetzgeber war also generell bewusst, dass der Gebrauch einer Sache zum natürlichen Verschleiß und einer erhöhten Mangelanfälligkeit führt. Diese Kriterien glaubte er, auf Tiere übertragen zu können. Bei Tieren besteht allerdings ein generelles Erkrankungs- und Verletzungsrisiko, welches schon mit ihrer Geburt und auch ohne Einwirkungsmöglichkeit ihres Züchters/Produzenten beginnt. Denn das Tier birgt von der Natur aus angelegte Verschlechterungs- und Veränderungsrisiken in sich. Es ist dem allgemeinen Lebensrisiko weitgehend schutzlos ausgeliefert, in dem es unsichtbare Krankheitserreger in seiner natürlichen Umwelt aufnimmt, ohne dass dies vom Züchter in diesem Moment bemerkt oder verhindert werden kann. Außerdem entfalten Tiere eigendynamisches Verhalten und können sich dadurch selbst Schaden zufügen, ohne dass dieses Risiko von ihrem Züchter ausgeschlossen werden könnte. Deshalb ist die Anwendung des Tatbestandsmerkmals eines "Gebrauchtseins" auf Tiere unter Sachmangelhaftungsgesichtspunkten ein gesetzgeberischer Fehlgriff.

Es ist allerdings für die Rechtsprechung problematisch, mit einer solchen Situation umzugehen, nachdem der Gesetzgeber die Unterscheidung von "neven" und "gebrauchten" Tieren ausdrücklich gefordert hat. Das Problem war schon vor Inkrafttreten des SMG erkannt worden, weshalb in der agrarrechtlichen Literatur schon seinerzeit gefordert wurde, diese Schwachstelle des Gesetzes ausdrücklich im Text auszuräumen (Adolphsen 2001; Pelhak 2001). Diese Hinweise hat der Gesetzgeber aber zunächst nicht registriert, wobei unklar bleibt, ob dies nur aus Unkenntnis der Problematik oder aus Bedenken gegen eine unvollständige Richtlinienumsetzung geschah.

Die Unterscheidungsnotwendigkeit zwischen "neven" und "gebrauchten" Tieren ist dann in der Literatur von Anfang an kritisiert worden (Westermann 2002), was teilweise in den Vorschlag mündete, die geforderte Unterscheidung einfach nicht vorzunehmen, indem nach einer "juristischen Sekunde" das Gebrauchtsein eines Tieres festgestellt wird (Vieweg und Vollkommer 2003; Brückner und Böhme 2002). Die Rechtsprechung ist allerdings zur Ignoranz gesetzlicher Vorgaben nicht befugt. Sie muss das Gesetz anwenden. Sie kann aber Regelungslücken schließen. Eine zu schließende Lücke könnte darin bestehen, dass der Gesetzgeber irrtümlich von einer sinnvollen, unter Risikogesichtspunkten vorzunehmenden Unterscheidungsmöglichkeit "never" und "gebrauchter" Tiere ausging, weil er glaubte, die Rechtsprechung habe hierfür bereits Kriterien herausgearbeitet, obwohl es gar keine Rechtsprechung zum Beginn des Gebrauchtseins im Gewährleistungsrecht gab. Die Rechtsprechung könnte deshalb die Tiere im Sachmangelhaftungsrecht analog einer gebrauchten Sache behandeln. Genauso ist nämlich seinerzeit der BGH in der vom Gesetzgeber in seinen Motiven genannten Rechtsprechung verfahren, als er Forellen nicht als "neu hergestellte Sache" bezeichnete, sondern sie nur aus den gewährleistungsrechtlichen Erwägungen des Einzelfalles wie eine solche behandelte (BGH 1986). Die Rechtsprechung sollte deshalb wegen tatsächlich nicht vorliegender Kriterien für gebrauchte Tiere und der Unmöglichkeit, solche Kriterien konkret zu fassen, die Tiere analog einer gebrauchten Sache behandeln.

Die jüngere Kommentarliteratur hat bereits prognostiziert, dass die vom Gesetzgeber irrtümlich angedachte Lesart der BGH-Rechtsprechung (BGH 1986), wonach bei lebenden Tieren zwischen "neu" und "gebraucht" zu unterscheiden sei, im Sachmängelhaftungsrecht des Verbrauchsgüterkaufs nicht zu halten sein wird und deshalb Tiere wie gebrauchte Sachen zu behandeln seien (Faust 2003; Lorenz 2004).

Die Viehkaufrechtsprechung hatte sich bisher nicht häufig mit dem Gebrauchtsein von Tieren zu befassen. Denn dieses Tatbestandsmerkmal spielt in erster Linie beim Auktionskauf und 
selten bei Veriährungserleichterungen eine Rolle. Letzteres gilt deshalb, weil auch beim Verkauf eines "gebrauchten Tieres" nur die Veriährung auf minimal ein Jahr erleichtert werden darf. Im Auktionskauf ist das Gebrauchtsein selten streitbefangen, weil viele Auktionspferde schon einem konkreten Verwendungszweck als Reitpferde zugeführt worden sind und deshalb auch tatsächlich gebraucht werden. Währenddessen sind Fohlen viel seltener als Reitpferde streitbefangen, weil sich eine eventuelle Verwendungszweckbeeinträchtigung häufig erst nach mehreren Jahren herausstellt, wenn diese Tiere nämlich geritten werden. Deshalb hat die Rechtsprechung sich bisher in erster Linie mit jungen Reitpferden befasst. Danach sind eine 4jährige, in Basisprüfungen siegreiche Stute (LG München I 2004) wie auch eine 4jährige gerittene Stute (LG Münster 2003), als "gebraucht" im Sinne von § 474 Abs. 1 Satz 2 BGB und eine 4 jährige, noch nicht tragende Zuchtstute nicht als "neu hergestellt" im Sinne von § $309 \mathrm{Nr}$. 8 a BGB (OLG Düsseldorf 2004) anzusehen.

\section{Beweislastumkehrregelung}

Nach dem Willen des deutschen Gesetzgebers können auch Tiere Gegenstand eines Verbrauchsgüterkaufs sein. Deshalb kommt auch die Anwendung der Beweislastumkehrregelung gem. § 476 BGB in Betracht, wonach von Gesetzes wegen zu vermuten ist, dass ein binnen sechs Monaten nach Übergabe aufgetretener Mangel auch schon zum Übergabezeitpunkt vorgelegen hat. Inzwischen ist höchstrichterlich klargestellt worden, dass dies eine lediglich in zeitlicher Hinsicht wirkende Vermutung beinhaltet (BGH 2004). Die Darlegungs- und Beweislast für die einen Sachmangel begründenden Tatsachen treffen auch nach Abnahme weiterhin den Käufer, so dass es nicht ausreicht, einen binnen sechs Monaten seit Gefahrübergang aufgetretenen Schaden zu behaupten. Vielmehr ergibt sich aus der Verpflichtung des Verkäufers, eine mangelfreie Kaufsache zu verschaffen, dass in der Annahme der Kaufsache durch den Käufer die Erfüllung dieser Verpflichtung zu sehen ist. Deshalb ist ein nach Übergabe der Kaufsache innerhalb von sechs Monaten auftretender Defekł zunächsł einmal lediglich ein Indiz dafür, dass dieser Schaden die Folge eines bei Gefahrübergang bereits vorliegenden Mangels sein könnte. Dies setz† aber voraus, dass es sich bei dem Defekt überhaupt um einen Sachmangel im Sinne von $\S 434$ BGB und nicht um einen auf fehlerhafter Handhabung, alters- und gebrauchsbedingtem Verschleiß oder ähnlichen Umständen beruhenden Schaden handelt. Erst ein vollständiger Beweis des Vorliegens eines Sachmangels kann die Beweislastumkehr auslösen (BGH 2004; OLG Bremen 2004; Lorenz 2004; Wietoska 2004).

Die klarstellenden Ausführungen des BGH über die den Sachmangel begründenden Tatsachen im Rahmen ergeben jedoch noch keine Anhaltspunkte dafür, ob Tiere überhaupt dem Verbrauchsgüterkaufrecht unterliegen oder ob sie zumindest aufgrund ihrer Art als Kaufsache oder der Art ihrer Mängel mit der Anwendung der verbrauchsgüterkaufrechtlichen Beweislastumkehrregelung unvereinbar sind.

\section{Art der Kaufsache}

Selbst wenn dem Käufer der Nachweis eines innerhalb der gesetzlichen Vermutungsfrist des $\S 476$ BGB aufgetretenen
Sachmangels gelingt, kommt die Beweislastumkehr trotzdem dann nicht in Betracht, wenn sie mit der Art der Kaufsache unvereinbar ist. Der Gesetzgeber hat sich allerdings vollständig darüber ausgeschwiegen, welche Arten von Kaufsachen er aus der Beweislastumkehrregelung ausgenommen wissen will und insbesondere, ob er damit Lebewesen und Naturprodukte meint. Er hat weder im Gesetzestext Beispiele genannt, noch in seinen Motiven genave Kriterien angegeben. Er hat lediglich angedeutet, dass gebrauchte Sachen wohl grundsätzlich für eine Ausnahme von der Beweislastumkehr in Betracht kommen (BT-Drs. 14/6040). Aufgrund dieser Andeutungen ist es der Rechtsprechung überlassen, den Ausnahmetatbestand mit Substanz zu füllen. Dieser Aufgabe widmet sich die Rechtsprechung verständlicherweise nur mit Zurückhaltung, weil man annehmen könnte, dass der Gesetzgeber den Tatbestand des $\S 476$ BGB sicherlich substantiierter formuliert hätte, wenn er Tiere oder bestimmte Tierarten von der Beweislastumkehrregelung hätte ausnehmen wollen. Diese Ansicht kann jedoch allenfalls auf den ersten Blick zutreffend sein. Denn der deutsche Gesetzgeber befand sich seinerzeit in dem Glauben, dass die Verbrauchsgüterkaufrichtlinie im europäischen Gesetzgebungsverfahren wirksam zustande gekommen sei und er deshalb zur vollständigen Umsetzung der Richtlinie verpflichtet sei, ohne die Möglichkeit zu haben, bestimmte Kaufsachen als Ausnahmetatbestand zu normieren. Dies scheint mit Hinsicht auf die bereits darstellten viehkaufrechtlichen Sonderregelungen im EU-Ausland eine Fehleinschätzung zu sein (Schwermaier 2003). Außerdem muss die Rechtsprechung bei der Schaffung von Kriterien für die Arten von Kaufsachen, die sich nicht für die Beweislastumkehrregelung eignen, berücksichtigen, dass den Gesetzgeber der bereits geschilderte Zeitdruck quälte und er erstmals eine tiermedizinische und agrarwissenschaftliche Beratung im Zuge eines viehkaufrechtlichen Gesetzgebungsverfahrens unterlassen hat. Beide Umstände könnten zu einer gewissen Oberflächlichkeit beigetragen haben. Dies wird u. a. dadurch deutlich, dass in der juristischen Literatur von Anfang an einhellig die Auffassung vertreten wird, dass Tiere sich für die verbrauchsgüterkaufrechtlichen Regelungen gar nicht (Westermann 2002), zumindest nicht für die Beweislastumkehrregelung eignen (Adolphsen 2001; Pelhak 2001; Bemmann 2003; Bruns 2004; a. A. v. Westphalen 2004; Oexmann und Wiemer 2004).

Greift man die Gedanken der jüngeren bürgerlichrechtlichen Kommentarliteratur auf, wonach eine Unterscheidung zwischen neven und gebrauchten Tieren sich prognostisch nicht halten wird, sondern Tiere generell analog einer gebrauchten Sache zu behandeln sein werden (Faust 2003; Lorenz 2004), ist anzunehmen, dass Tiere vermehrt aufgrund ihrer Art als Kaufsache nicht Gegenstand der Beweislastumkehrregelung sein werden. Dies zeichnet sich schon $a b$, wenn man einen Blick auf die juristische Literatur zum Kfz-Handel wagt. Denn auch dort stellt sich die Frage, ob gebrauchte Fahrzeuge aufgrund ihrer Art teilweise von der Beweislastumkehrregelung auszunehmen sind. Dies soll jedenfalls immer dann der Fall sein, wenn keine gesicherten Statistiken vorliegen, anhand derer eine retrospektive Überprüfung vorgenommen werden kann (Wietoska 2004). Wendet man diese Gedanken auf Tiere an, führt dies zur Ausnahme aller Lebewesen aus der Beweislastumkehrregelung. Denn es gibt keine Sicherheit über die Beschaffenheit eines Tieres, weil es für Tiere keine Konstruktionspläne gibt und insbesondere der Mensch in die 
Gestaltung der Tiere nicht eingreifen kann. Es handelt sich jeweils um Unikate, die die Natur geschaffen hat. Sie sind allein schon deshalb für die Beweislastumkehrregelung ungeeignet, weil der Verbraucherschutzgedanke der Beweislastumkehrregelung beim Verkauf von Tieren nicht greift. Denn der Pferde kaufende Verbraucher ist regelmäßig kein schutzbedürftiger, in rechtlicher und hippologischer Unkenntnis handelnder Vertragspartner. Vielmehr handelt es sich regelmäßig um einen kenntnisreichen Tierhalter und Reitsportler, der die Vorteile des Verbrauchsgüterkaufrechts in nun einer Weise ausnutzen kann, die sich schädlich auf die Tierzucht auswirkt. Dadurch ist die Branche derzeit von der Angst besessen, sich ein bis zwei Jahre in der Gefahr zu befinden, jedes Tier aus Gründen zurücknehmen zu müssen, die zum Verkaufszeitpunkt nicht ansatzweise kalkulierbar sind. Ein solchermaßen die Wirtschaft lähmendes Gesetz läuft seinem Zweck zuwider.

Der Verbraucher ist nach juristischer Beratung aufgrund der ihm günstigen Gesetzeslage derzeit bemüht, dem Landwirt, Züchter oder Händler ein Pferd möglichst per Handschlag gegen bloße Zahlungsquittung abzukaufen und eine in den Kaufvertrag eingebundene tierärztliche Untersuchung sowie den Abschluss einer Beschaffenheitsvereinbarung zu umgehen, um zunächst einmal über einige Wochen zu prüfen, ob er mit seinem Pferd harmoniert und erfolgreich am Sport teilnehmen kann. Erfüllen sich solche Erwartungen nicht, begibt er sich innerhalb der gesetzlichen Vermutungsfrist auf Fehlersuche, indem er sein Pferd solange röntgt oder per Ultraschall und Szintigraphie untersuchen lässt, bis eine Abweichung vom physiologischen Normbild gefunden ist. Diese wird dann zum Anlass einer Sachmangelrüge erhoben und die vom deutschen Gesetzgeber völlig fehleingeschätzten tiermedizinischen Möglichkeiten, den gerügten Tatbestand retrospektiv über mehrere Monate zu bewerten (Eversfield 2004), sollen dann dazu verhelfen, dass sich diese Unsicherheiten zum Nachteil des Unternehmerverkäufers auswirken. Diesem Dilemma kann ein Verkäufer entgegen vereinzelt vertretener Auffassung auch dann nicht entgehen, wenn die tiermedizinischen Standards der Kaufuntersuchungen erhöht werden und jeder Verkäufer eine diesem Standard entsprechende Untersuchung durchführt (Wiemer und Oexmann 2004; v. Westphalen 2004). Denn es bliebe dem Käufer stets die Möglichkeit, sich im Falle der Kaufreue außerhalb des Kaufuntersuchungsspektrums auf Fehlersuche zu begeben oder die Genetik als Mangel zu rügen. Dabei haben sich schon richtige Modemängel entwickelt. Dies führt sogar zu Missbrauch, indem Abweichungen vom physiologischen Normbild gerügt werden, die eine Vielzahl von Pferden aufweisen, die gleichwohl zeitlebens hervorragende sportliche Leistungen bringen. In diesem Zusammenhang ist insbesondere das Kissing-Spines-Syndrom seit der Schuldrechtsreform zum "Mangel der Wahl" geworden (Plewa 2003; Ende 2004).

Vor diesem Hintergrund zeichnet sich in der Rechtsprechung eine Tendenz ab, Tiere als Kaufsache aufgrund ihres unter Ausschluss menschlicher Einflüsse erworbenen Unikatstatus und ihres verfassungsrechtlichen Schutzes aus der Beweislastumkehrregelung generell auszuschließen (AG Helmstedt 2003; LG Braunschweig 2003). Insbesondere gebrauchte Pferde sind aufgrund ihrer Art und im Hinblick auf die schnellen Veränderungsmöglichkeiten ihres Allgemein- und Gesundheitszustandes kaum für die Beweislastumkehrregelung geeignet (a) OLG Oldenburg 2004). So spricht alles dafür, dass Pferde aufgrund ihrer Art als Kaufsache für die Beweislastumkehrregelung ungeeignet sind (b)OLG 2004), wobei häufig dahingestellt bleiben kann, ob nun allein schon aufgrund der Art der Kaufsache oder zumindest aufgrund der Art des Mangels die Beweislastumkehrregelung ausscheidet. Jedenfalls geht die Rechtsprechung mit zunehmender Regelmäßigkeit davon aus, dass bereits die Art der Kaufsache "Pferd" die Anwendung der Beweislastumkehrregelung fraglich erscheinen lässt (LG Aurich 2004; LG Verden 2004; LG Lüneburg 2004; LG Flensburg 2004).

\section{Art des Mangels}

Sogar der Gesetzgeber hat erkannt, dass häufig Tierkrankheiten aufgrund der Art des Mangels mit der Beweislastumkehr unvereinbar sind, weil aufgrund ungewisser Inkubationszeiten unsicher bleibt, ob sich ein Tier bereits vor oder erst nach Lieferung angesteckt hat. Diese Unsicherheit eignet sich dann nicht für eine gesetzliche Vermutung (BT-Drs. 14/6040). Leider hat der Gesetzgeber seiner weisen Erkenntnis in den Motiven den unsäglichen Nachsatz angefügt, dass die Ungeeignetheit für eine Beweislastumkehr nicht unbedingt auch in Bezug auf andere Tiermängel gelten muss. Eine Begründung dieser ohne Sinnzusammenhang an den Text der Motive angefügten These hat er unterlassen, obwohl eigentlich allgemein bekannt ist, dass fast alle Verletzungen durch plötzliche äußere Einwirkung von einem Moment zum anderen entstehen und deshalb noch weniger als ansteckende Krankheiten retrospektiv bewertet werden können. Ebenso gibt es eine Vielzahl degenerativer Erkrankungen, für die es mangels Ansteckung keine Inkubationszeiten gibt. Dennoch ist bekannt und wissenschaftlich belegt, dass auch diese Erkrankungen innerhalb weniger Wochen, in jedem Fall aber in Zeiträumen entstehen können, die erheblich kürzer als die gesetzliche Vermutungsfrist sind (Eversfield 2004).

Es bleibł völlig unklar, warum der Gesetzgeber sich nicht zu Erkrankungen und Verletzungen geäußert hat, die zwar nicht durch Erreger übertragen werden, aber innerhalb von kurzen Fristen entstehen können und deshalb in gar keinem Verhältnis zur 6-Monatsfrist des $\S 476$ BGB stehen.

Darüber hinaus gibt es noch das weite Spektrum der Verhaltensauffälligkeiten, die aufgrund eines meist unbekannten oder unerkannten Initialtraumas von einem Moment zum anderen entstehen können. Es ist unvorstellbar, dass solche Erscheinungen für irgendeine rückwirkende Betrachtung geeignet sein könnten.

Letztendlich schweigt der Gesetzgeber sich auch völlig über die vielen Rittigkeitsprobleme und Schwierigkeiten im Umgang mit dem Tier aus, die ein Käufer häufig mit seinem neven Gefährten erlebt und als deutliche Gebrauchsbeeinträchtigung empfindet. Hierfür gibt es weder Inkubationszeiten, noch sind es Umstände, die eindeutig oder gar ausschließlich der Sphäre des Tieres zuzuordnen sind. Folglich ist nicht nur eine auf den Übergabezeitpunkł zurückbezogene Feststellung unmöglich. Vielmehr bereitet schon die Feststellung des Sachmangeltatbestandes Probleme. Denn der Käufer müsste darlegen und beweisen, dass er reiterlich und im sonstigen Umgang mit dem Pferd alles richtig gemacht hat und es auch ansonsten keinen Einflüssen ausgesetzt hat, die 
zur Veränderung führen könnten. Nach einem regelmäßig vor jedem Pferdekauf erfolgreich durchgeführten Proberitt ist es nahezu unmöglich, eine Rittigkeitsproblematik schlüssig als Sachmangel darzulegen.

Zusammenfassend lässt sich feststellen, dass nahezu alle Mängel eines Pferdes nicht über einen Zeitraum von sechs Monaten retrospektiv bewertet werden können, solange es keine Anknüpfungstatsachen aus der Sphäre des Verkäufers wie z. B. alte Röntgenbilder - gibt. Trotzdem hat sich der Gesetzgeber ohne ein Wort der Begründung zu dem Satz hinreißen lassen, dass die Unvereinbarkeit eines Mangels mit der Beweislastumkehrregelung nicht unbedingt für andere Fehler eines Tieres gilt. Es ist bisher nicht zu ergründen, ob ein Gedanke und gegebenenfalls welcher Gedanke dieser Formulierung zugrunde lag.

Die gängige Kommentarliteratur konnte sich erwartungsgemäß zunächst einmal nur darauf beschränken, die Begründungen des Regierungsentwurfs wiederzugeben (Lorenz 2004; Putzo 2005; Faust 2003; Schmidt-Räntsch 2002), so dass es auch insoweit vollständig der Rechtsprechung überlassen bleibt, die Kriterien zur Frage einer retrospektiven Bewertbarkeit für die weit überwiegende Zahl derjenigen Tiermängel zu entwickeln, die nicht durch Ansteckung entstehen. Dadurch hat der Gesetzgeber zunächst einmal eine große Rechtsunsicherheit und auch eine Flut von Viehmängelprozessen einer besonderen Art verursacht, die vor der Schuldrechtsreform undenkbar gewesen wären. Es ist nämlich zahlreich versucht worden, den unlieb gewordenen Sportkameraden "Pferd" über die "Segnungen" des Verbrauchsgüterkaufrechts immer dann wieder beim Züchter abzuladen, wenn die Reiterei nicht funktionierte oder aus anderen Gründen der erhoffte sportliche Erfolg ausblieb. Mittlerweile zeichnen sich jedoch ganz deutliche Tendenzen in der Rechtsprechung $a b$, wonach dies nicht erwünscht ist.

Dies ist am eindeutigsten bei den sog. Rittigkeitsmängeln der Fall. Die Rechtsprechung erkennt, dass allein schon der mit dem Verkauf einhergehende Wechsel im Reitstil zu veränderten Reaktionen des Pferdes führen kann und folglich mangelnde Rittigkeit und Beherrschbarkeit eines Pferdes keine Umstände sind, mit denen eine Beweislastumkehr vereinbar wäre (LG Aurich 2004). Dies gilt auch deshalb, weil ohnehin nicht davon ausgegangen werden kann, dass Rittigkeit und Einsetzbarkeit im Turniersport einem Pferd daverhaft und unabhängig vom jeweiligen Reiter anhaften. Diese Erkenntnis lässt es auch zweifelhaft erscheinen, ob die Rittigkeit überhaupt als ein in der Sache selbst begründeter Umstand anzusehen ist. Mithin steht bereits die Tatbestandsmäßigkeit im Sinne von $\S 434$ BGB in Frage (OLG Oldenburg 2004 a). Rittigkeitsprobleme erfüllen insbesondere nicht den Sachmangeltatbestand und erst recht nicht die Voraussetzungen für eine Beweislastumkehr, wenn das Pferd grundsätzlich geeignet ist, aber nicht unter jedem Reiter und insbesondere nicht unter seinem Käufer problemlos geht (LG Hildesheim 2004). Teilweise wird die Rittigkeit auch als eine vornehmlich in der Art der Betreuung anzusiedelnder Umstand angesehen, weshalb aus diesem Grunde die Beweislastumkehr nicht eingreifen darf (LG Lüneburg 2003). Noch zurückhaltender verhält sich die Rechtsprechung bei der Anwendung der Beweislastumkehr auf Rittigkeitsprobleme im Sinne aus- bleibender Springsportleistungen. Denn auch insoweit gilt, dass eine weigerliche Einstellung des Pferdes zum Springsport deutlich trainings- und reiterabhängig sein kann und schon deshalb für die Anwendung von $\S 476$ BGB nicht in Betracht kommt. Dies gilt insbesondere, wenn das Pferd unter dem Verkäufer Erfolge erzielte und der Proberitt zufrieden stellend ausfiel. Dies gilt aber auch dann, wenn sich nach Leistungsverweigerung unter dem Käufer selbst bei späterer Inanspruchnahme professioneller Hilfe keine Erfolge wieder einstellen (LG Lüneburg 2004). Auch die völlige Leistungsverweigerung eines S-Springpferdes unter einem Reiter, der von sich behauptet, der erfolgreichste seiner Sparte zu sein, ist weder für eine Beweislastumkehr geeignet, noch erfüllt sie den Sachmangeltatbestand. Dies gilt jedenfalls, sofern das Pferd jedenfalls vor Übergabe Erfolge in S-Springen errang. Denn ein künftiger und auch unter dem Käufer zu erzielender Sporterfolg wird nicht geschuldet (AG Hannover 2004; LG Hannover 2004). Selbst wenn die Kaufvertragsparteien übereinstimmend ein Springpferd als für Klasse M geeignet bezeichnet haben, liegt kein Sachmangel vor und besteht erst recht kein Raum für die Anwendung von $\S$ 476 BGB, wenn das Pferd sein vorgegebenes Ausbildungsziel nicht erreicht. Denn es steht von vornherein fest, dass das Gelingen einer Ausbildung und die anschließende erfolgreiche Teilnahme am Springsport mit Unwägbarkeiten behaftet sind (OLG Frankfurt 2004).

Ähnlich bewertet die Rechtsprechung die Verhaltensauffälligkeit des Webens, die von Tiermedizinern nicht ohne Kenntnis des ursächlich gewordenen Initialtraumas rückwirkend beurteilt werden kann und aus tiermedizinischer Sicht ohnehin nicht erheblich ist, weil nachweislich keine Gesundheitsund/oder Leistungsbeeinträchtigung einhergeht (Eikmeyer 1990; Köhler und Kraft 1984; Pick et al. 2003; Dietz und Huskamp 1999; Wintzer 1982). Weben ist deshalb eindeutig für eine Anwendung der Beweislastumkehr ungeeignet (LG Aurich 2004; OLD Oldenburg 2004 b). Die Verhaltensauffälligkeit des Koppens soll beim Reit- und Springpferd gar nicht als Mangel anzusehen sein, weil sie sich auf die Eignung eines Reit- und insbesondere Springpferdes nicht auswirkt, solange keine gesundheitlichen Folgeerscheinungen nachweisbar sind (LG Bückeburg 2004).

Auch Borreliose sowie eine Hefepilzerkrankung des Darmtraktes können die Umkehrregelung gem. § 476 BGB nicht auslösen; denn selbst wenn man zugunsten des Käufers seinen Vortrag über Inkubationszeiten von einigen Wochen als wahr unterstellt, ist für die Anwendung einer Vermutung über einen Zeitraum von mehreren Monaten kein Raum (AG Osterholz-Scharmbeck 2003).

Die Anwendung von $\S 476$ BGB auf einen Tumor ist fraglich, kann aber natürlich dann dahinstehen, wenn konkrete Anhaltspunkte für das Vorliegen im Übergabezeitpunkt vorhanden sind (AG Lemgo 2004).

Kehlkopfpfeifen und Follikelkatarrh sind aufgrund ihrer Art als Sachmangel nicht für eine Beweislastumkehr geeignet. Dies folgt für das Kehlkopfpfeifen allein schon aus dem Umstand, dass der frühere Gesetzgeber unter tiermedizinischer Beratung nur eine Gewährfrist von 14 Tagen annahm, so dass sich die Anwendung einer 6-monatigen Frist verbietet (AG Hannover 2004). 
Erkrankungen des Bewegungsapparates, die typischerweise verletzungsbedingt auftreten, sind ungeeignet für die Anwendung von $\S 476$ BGB. So kann z. B. der Fesselträgerschaden bereits durch einen Fehltritt entstehen, wenn es dabei zu Zerreißungen der Sehnenfasern kommt (LG Neubrandenburg 2004).

Auch viele röntgenologisch darstellbare Veränderungen am Knochen sind ungeeignet für die Beweislastumkehr. So gilt für viele Röntgenbefunde im Sinne von Spat, dass diese schon nicht den Sachmangeltatbestand erfüllen, wenn sie sich nicht als ursächlich für eine wiederkehrende Lahmheit erweisen. Selbst eine Lahmheit, die ursächlich auf dem Röntgenbefund beruht, lässt sich nicht über Zeiträume von mehr als sechs Wochen retrospektiv bewerten (AG Bad Gandersheim 2005; LG Lüneburg 2005; LG Paderborn 2004; OLG Hamm 2005).

Gleiches gilt im Grundsatz für das Kissing-Spines-Syndrom, weil regelmäßig Röntgenbefunde vorliegen, die zahlreiche Pferde in einem gleichen oder gar stärkeren Ausmaß zeigen, wobei diese Pferde beständig Leistungen im Spitzensport erbringen, so dass der bloße Röntgenbefund nur schwerlich den Sachmangeltatbestand erfüllen kann. Dies wird durch die jüngste Studie über Röntgenuntersuchungen an den Dornfortsätzen von 2-bis 6-jährigen nicht gerittenen bzw. wenig gerittenen Warmblutpferden bestätigt. Von 900 untersuchten Pferden, die keine klinischen Befunde aufwiesen, zeigten 67,5 \% der Tiere Zubildungen unterschiedlicher Graduierung bis hin zu sich überlappenden Dornfortsätzen. Ein Vergleich mit einer Gruppe von 176 Pferden, die klinische Rückenbefunde aufwiesen, ergab, dass diese Gruppe die gleiche statistische Befundhäufigkeit und-ausprägung zeigte, wie die Gruppe der Pferde ohne klinischen Befund (Brunken 2005; Gerhards und Ranner 2002; Erichsen et al.). Das stellt jegliche Bedeutung des Röntgenbefundes an den Dornfortsätzen zunächst in Frage. Deshalb ist ein Mangel regelmäßig nur dann anzunehmen, wenn ein Ursachenzusammenhang zwischen dem Röntgenbefund und einem klinischen Befund nachweisbar ist. Solches kann regelmäßig nur mit aufwendiger Differenzialdiagnose festgestellt werden. Außerdem lässt sich die klinische Symptomatik eines Kissing-Spines-Syndroms selten retrospektiv über den Zeitpunkt ihres ersten Auftretens hinausgehend bewerten (LG Potsdam 2003; LG Hannover 2004), so dass eine Beweislastumkehr nicht in Betracht kommt.

Fraglich ist, ob allergische Geschehen wie z. B. das Sommerekzem sich für eine Beweislastumkehr eignen, solange keine Anknüpfungspunkte für eine in der Sphäre des Verkäufers aufgetretene Erkrankung feststellbar sind. Es ist unklar, ob solche Allergien im Verlauf des Lebens erworben werden oder eine genetische Prädisposition besteht, die beim Hinzutreten weiterer, außerhalb der Genetik liegender Umstände zur Ausprägung einer Erkrankung führt, indem sich klinische Symptome zeigen. Selbst wenn eine genetische Prädisposition vorliegen sollte, nimmt die Rechtsprechung bisher keine Eignung für die Beweislastumkehr gem. § 476 BGB an, weil die genetische Prädisposition für sich genommen keinen Mangel darstellt, sondern jedes Pferd mit dieser Prädisposition lebenslang ohne Krankheitsausbruch bleibt, solange nicht die weiteren zur Ausprägung des Mangels erforderlichen Umstände irgendwann komplett hinzutreten (LG Arnsberg 2004; LG Lüneburg 2005; Vieweg und Vollkommer 2003).
Die Diskussion über die Anwendung der Beweislastumkehrregel auf einen Mangel, der eine gewisse genetische Komponente haben könnte oder gar aufweist, macht allzu deutlich, dass die Beweislastumkehrregelung generell für Tiere ungeeignet ist. Denn wollte man die anteilige genetische Prädisposition eines Mangels für sich genommen auch schon als Mangel ansehen, wäre jedes Lebewesen mangelhaft. Jeder Kaufvertrag über ein Tier wäre dann innerhalb der Verjährungsfrist nach Belieben des Käufers rückabwickelbar. Es ist deshalb abzulehnen, die Genetik eines Tieres für sich genommen dem Mangeltatbestand zu unterziehen. Denn für fast alle Symptome gibt es genetische Veranlagungen. Dies würde zu grotesken Ergebnissen führen, die man am Beispiel des Melanoms besonders deutlich machen kann. Es entspricht dem derzeitigen Stand der tiermedizinischen Wissenschaft, dass vorzugsweise Schimmel am Melanom erkranken und dann im Alter bis zu $80 \%$ aller Schimmel rasseunabhängig Melanome aufweisen (Dietz und Wiesner 1982; Wintzer 1982; Dietz und Huskamp 1999), so dass die Schimmelfarbe als genetische Prädisposition für die Neigung zur Erkrankung am Melanom gilt. Würde man nun die genetische Prädisposition für sich genommen bereits als Mangel ansehen, wäre jeder Schimmel ein Mangel und jeder Schimmelkauf rückabwickelbar. Solche Beispiele ließen sich beliebig fortführen und zeigen eindrucksvoll, dass die Einbeziehung des Tierkaufs in das allgemeine Kaufrecht und insbesondere in das Verbrauchsgüterkaufrecht keine gesetzgeberische Errungenschaft ist, sondern zu unlösbaren Problemen führt. Deshalb ist der Ruf nach der Schaffung eines Tierkaufrechts im BGB und der neidvolle Blick auf diejenigen EU-Mitgliedsstaaten, die es bei gleichzeitiger Umsetzung der Verbrauchsgüterkaufrichtlinie geschafft haben, sich ein Viehkaufrecht zu erhalten, nur allzu verständlich.

\section{Fazit}

Es ist festzustellen, dass die Rechtsprechung mit zunehmender Tendenz nahezu alle Tiermängel, deren Entstehungszeit nicht sicher retrospektiv beurteilt werden kann, aus der verbrauchsgüterkaufrechtlichen Beweislastumkehrregelung ausnimmt. Es bleibt abzuwarten, ob die Entwicklung in dieser Richtung fortschreitet. Währenddessen ist noch unklar, ob die Wirksamkeit der Verbrauchsgüterkaufrichtlinie im Hinblick auf ihre Anwendung im Tierkaufrecht durch den EuGH überprüft wird. Bisher ist nicht bekannt, ob derartige Verfahren anhängig sind. In der Praxis ist festzustellen, dass sich die Notwendigkeit einer Anrufung des EuGH zur Klärung dieser Frage bisher noch nicht gestellt hat, weil die Beweislastumkehrregelung wegen der Art des Mangels oder der Art der Kaufsache regelmäßig nicht angewandt wird, so dass es möglicherweise noch sehr lange davert, bis die Nichtigkeitseinrede eines Unternehmers zur Anrufung des EuGH führt.

\author{
Literatur \\ Adolphsen J. (2001): Die Schuldrechtsreform und der Wegfall des \\ Viehgewährleistungsrechts, AgrarR 169-174 \\ AG Bad Gandersheim (2005): Urt. v. 23.04.2004 in DPT 41 \\ AG Hannover (2004): Urt. v. 29.10.2004 Az: 445 C 7290/04 \\ AG Helmstedt (2003): Urt. v. 01.04.2003 Az: 3 C 486/02 \\ AG Lemgo (2004):Urt. v. 06.10.2004 Az: 512 C 5538/04
}


AG Osterholz-Scharmbeck (2003):Urt. v. 22.09.2003 Az: 13 C 268/03

Bemmann K. (2003): Der Pferdekauf im Jahr nach der Schuldrechtsreform, AgrarR 233-238

BFH (1981): Urt. v. 23.07.1981 BS+BI II, 672

BFH (1989): Urt. v. 09.12.1988 BStBI II, 244

BFH (1997): Urt. v. 15.05.1997 BStBI II, 575-578

BGH (1986): Urt. v. 03.07.1985 in NJW-RR, 52-54

BGH (2004): Urt. v. 02.06.2004 in NJW, 2299-2301

Brückner S. und Böhme A. (2002): Neues Kaufrecht - Wann ist ein Tier gebraucht. in MDR, 1406-1409

Brunken G. (2005): Radiologische Untersuchungen an den Dornfortsätzen der Brustwirbel - Neue Erkenntnisse? Vortrag vor der Akademie für tierärztliche Fortbildung am 22.01.2005

Bruns E. (2004): Zucht und Haltlung von Sportpferden, 55-97

Däubler-Gmelin H. (2001): Die Entscheidung für die sog. Große Lösung bei der Schuldrechtsreform. NJW, 2281-2289

Dauner-Lieb B. (2004): Drei Jahre Schuldrechtsmodernisierungsgesetz. AnwBI., 577

Dennhardt (2003): in Bamberger H. G. und Roth H., Kommentar zum BGB

Dietz O. und Wiesner E. (1982): Handbuch der Pferdekrankheiten für Wissenschaft und Praxis, 348

Dietz O. und Huskamp B. (1999): Handbuch Pferdepraxis, 240-241, 712,776

Eikmeyer H. (1990): Lehrbuch der gerichtlichen Tierheilkunde

Ende H. (2004): Forensische Probleme in der Pferdepraxis, bptinfo 9, $14-15$

Erichsen C., Esksell P., Roethlisberger K., Lord P. und Johnston C. (2004): Relationship between scintigraphic and radiographic evaluations of spinous processes in the thoracolumbar spine in riding horses without clinical signs of back problems, Equine vet. J. 36, 458-465

Eversfield S. (2004): Fazit: Zwei Jahre Schuldrechtsreform in bptinfo, 19-20

Faust F.(2003): in Bamberger und Roth Kommentar zum BGB 1. Aufl. München, §§ 474, 476

Fuchs S. (2004): Die Neue Pferdestärke in DIE ZEIT Nr. 35, 24-25

Gerhards H. und Ranner W. (2002): Vorkommen und Bedeutung von Rückenerkrankungen - insbesondere des "Kissing-Spine"-Syndroms - bei Pferden in Süddeutschland, Pferdeheilkunde 19, 21 33

Grimm C. (2004): Agrarrecht 2. Aufl. 119-124

Heinrichs H. (2005): In Palandt, O. Bürgerliches Gesetzbuch 64. Aufl., 383

Kamph H. (2004): Über 300 Jahre Tradition in Der Hannoveraner 8, 43

Köhler H. und Kraft H. (1984): Gerichtliche Veterinärmedizin, 200

Kreling K. (2004): Kleiner Nachbar - große Ideen in Pferdesport International 25, 36-38

LG Arnsberg (2004): Urt. v. 06.02.2004 Az: $4 \bigcirc 396 / 02$

LG Aschaffenburg (1990): Urt. v. 14.12.1989 in NJW S. 915-916

LG Aurich (2004a): Beschl. v. 17.03.2004 Az: $2 \bigcirc 54 / 04$

LG Aurich (2004b): Beschl. v. 26.02.2004 Az: 2 O 54/04

LG Aurich (2004c): Urt. v. 24.02.2004 Az: 3 ○ 256/04

LG Braunschweig (2003): Beschl. v. 18.06.2003 Az: 4 S 218/03

LG Flensburg (2004): Urt. v. 16.07.2004, Az: 2 O 328/03

LG Hannover (2004): Urt. v. 21.09.2004 Az: 17 O 293/02

LG Hannover (2004): Beschl. v. 22.12.2004 Az: 13 S 94/04

LG Hildesheim (2004): Urt. v. 30.12.2004 Az: 2 O 70/04

LG Lüneburg (2003): Urt. v. 18.11.2003 Az: 4 ○ 286/03

LG Lüneburg (2004a): Urt. v. 16.03.2004 Az: 4 ○ 322/03

LG Lüneburg (2004b): Urt. v. 16.03.2004 Az: 4 O 347/03

LG Lüneburg (2005): Urt. v. 16.03.2004, DPT S. 42

LG München I (2004): Urt. v. 09.09.2004 Az: $26 \bigcirc$ 1240/02
LG Münster (2003): Urt. v. 19.03.2003 Az: 4 O 518/02

LG Neubrandenburg (2004): Urt. v. 07.05.2004 Az: 3 ○ 565/03

LG Oldenburg (2004): Beschl. v. 05.02.2004 Az: 9 O 3147/03

LG Paderborn (2004): Urt. v. 31.03.2004 Az: 4 O 512/03

LG Potsdam (2003): Urt. v. 19.08.2003 Az: 6 ○ 328/02

LG Verden (2004): Beschl. v. 24.03.2004 Az: 2 S 394/03

Lorenz S. (2004): in Münch. Kommentar zum Bürgerlichen Gesetzbuch 4 . Aufl.

Lorenz S. (2004): Sachmangel und Beweislastumkehr im Verbrauchsgüterkauf - zur Reichweite der Vermutungsregelung in $\S$ 476 BGB in NJW, 3020-3022

Oexmann B. und Wiemer N. (2004): Beweislastumkehr des § 476 BGB im Rahmen des Pferdekaufes. Pferdeheilkunde 20, 368-371

OLG Bremen (2004): Urt. v. 11.03.2004 in OLGR 319-320

OLG Düsseldorf (2004): Urt. v. 02.04.2004, ZGS 271-275

OLG Frankfurt a. M. (2004): Urt. v. 19.04.2004 Az: 17 U 4/04

OLG Hamm (2005): Urt. v. 15.10.2004, DPT S. 41

OLG Oldenburg (2004a): Beschl. v. 11.05.2004 Az: 8 W 76/04

OLG Oldenburg (2004b): Urt. v. 17.06.2004 Az: 14 U 42/04

OVG Schleswig (2004): Unt. v. 04.11.2003, RdL S. 51-52

Pelhak J. (2001): Stellungnahme des Ausschusses für Tierzuchtrecht der deutschen Gesellschaft für Agrarrecht zum Entwurf eines Gesetzes zur Modernisierung des Schuldrechts. AgrarR, 312-313

Pick M., v. Salis B. und Schüle E. (2003): Liste zur Beurteilung von Minderungen des Verkehrswertes eines Pferdes, 3

Plewa D. (2003): Neues Pferdekaufrecht. Eine erste Bilanz - Nichts ist unmöglich. in St. Georg 7,40

Putzo H. (2005): In Palandt O., Bürgerliches Gesetzbuch 64. Aufl. $\S 476$

Redeker K. (2004): Wege zu besserer Gesetzgebung, ZRP S. 160163

Reischaver R. (2002): Das Gewährleistungsrecht und seine schadenersatzrechtlichen Folgen. JBI. ,137-204

Schermaier M. J. und Saenger I. (2003): Verbraucherkauf in Europa 191-208

Schmidt-Räntsch J. (1998): Zum Stand der Kaufrechtsrichtlinie, ZIP 849-853

Schmidt-Räntsch J. (2002): Das neve Schuldrecht 299

Schulze R. und Schulte-Nölke H. (2001): Die Schuldrechtsreform vor dem Hintergrund des Gemeinschaftsrechts, 1. Aufl.

Stampf O. und Stuppe A. (2002): Pferde zu Maschinen gemacht in Der Spiegel 8, 190-192

Vieweg K. und Vollkommer G. (2003): Spektrum des Sportrechts $391-412$

Vollkommer G. (2003): Das neue Recht beim Tierkauf in Pferdespiegel 12, 10-13

Wenzel J. (2003): in Münchn. Kommentar zum Bürgerlichen Gesetzbuch 4. Aufl.

Wertenbruch J. (2004): Gewährleistung beim Verkauf von Kunstgegenständen nach nevem Schuldrecht NJW, 1977-1982

Westermann H. (2002): Das neve Kaufrecht in NJW, 241-253

Westphalen v. E. (2004): Die Beweislastumkehr zugunsten des Pferdekäufers nach § 476 BGB, ZGS, 341-344

Wietoska E. (2004): Die Beweislastumkehr in $\S 476$ BGB in der Praxis des Gebrauchtwagenkaufs in ZGS, 8-12

Wintzer H.-J. (1982): Krankheiten des Pferdes, 483

RA Kai Bemmann

Kanzlei Berner, Fischer und Partner

Burgwall 2

27283 Verden

bemmann@bernerfischer-partner.de 